\title{
Time and charge characterization of Hamamatsu Photonics sili- con photomultipliers
}

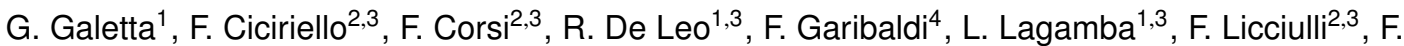

 \\ ${ }^{1}$ Dipartimento Interateneo di Fisica "M. Merlin", Bari, Italy \\ ${ }^{2}$ DEE, Politecnico di Bari, Bari, Italy \\ ${ }^{3}$ Istituto Nazionale di Fisica Nucleare, Sezione di Bari, Bari, Italy \\ ${ }^{4}$ Istituto Nazionale di Fisica Nucleare, Sezione di Roma, Roma, Italy \\ ${ }^{5}$ Istituto Nazionale di Fisica Nucleare, Sezione di Lecce, Lecce, Italy
}

\begin{abstract}
Two Hamamatsu Photonics silicon photomultipliers (SiPM) with same cell size $50 \times 50 \mu^{2}$ and different sensitive area $\left(1 \times 1 \mathrm{~mm}^{2}\right.$ vs. $\left.3 \times 3 \mathrm{~mm}^{2}\right)$ have been characterized in charge and time by means of a $409 \mathrm{~nm}$ pico-laser. The time resolution was studied for the detection of few photoelectrons. The results obtained are relevant for the employment of SiPM in ring imaging Cherenkov detectors (RICH).
\end{abstract}

\section{Introduction}

SiPM or Multi-Pixel Photon Counters (MPPC) have increasingly gained interest over ordinary photomultipliers because of several advantages. The low-voltage operation, small sizes, single photon counting capabilities, magnetic field immunity and others make useful their employment in many experiments, spanning from medical imaging to high energy particle calorimetry. In particular, their efficient single photon counting, short signal rise time and small size make SiPM/MPPC suitable [1] for high granularity Cherenkov ring imaging detectors. We have performed a characterization of time and charge of two models [2] of Hamamatsu MPPC (Fig. 1a) and report the results in this contribution.

\section{Experimental set-up}

MPPC are known [3] to perform very well in timing measurements owing to their typical signal shape (Fig. 1a), having short rise time. As a matter of fact, the timing performance is strongly influenced by the front-end electronics bandwidth. For the present measurements a $63 \times$ pre-amplifier/shaper board based on the scheme suggested by Hamamatsu was used. Amplification is mandatory in the detection of a low average number of photoelectrons ( $p e$ 's).

The measurement set-up (Fig. 1b) is based on a pulsed light source and MPPC sensor. The light source was a pico-laser [4] PIL040SM by Advanced Laser Diode Systems Berlin, emitting pulses at a wavelength of $\lambda=409 \pm 1 \mathrm{~nm}, \sigma=20$ ps duration, and operated by an external trigger at $25 \mathrm{~Hz}$.

\footnotetext{
a e-mail: roberto.perrino@le.infn.it
} 


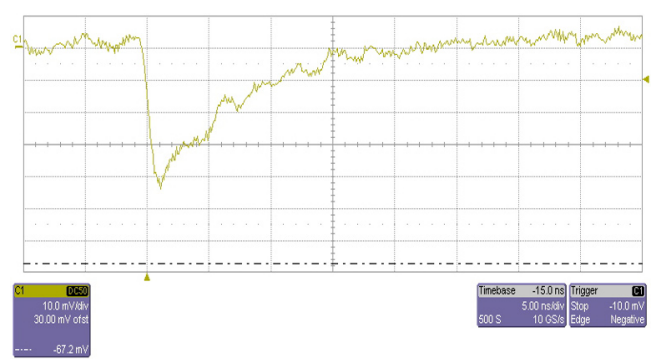

(a)

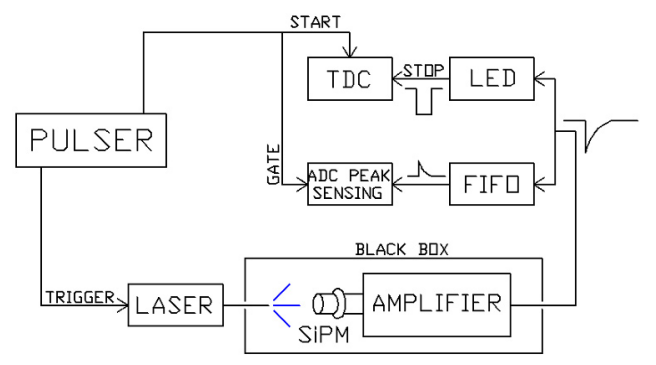

(b)

Figure 1: (a) Digitized signal from 1-pe excitation in the 400 cells device $(10 \mathrm{mV} / \mathrm{div}, 5 \mathrm{~ns} / \mathrm{div}, 10$ $\mathrm{GSa} / \mathrm{s}$ ). (b) Sketch of the measurement setup.

The light source was directed to the MPPC, enclosed in a lightproof box, through fiber optics. The signal from the front-end board was subsequently split in order to get time and charge simultaneous measurements. Charge was obtained by a Peak Sensing ADC Ortec AD811 (1 mV/channel), and time was derived in a leading edge discriminator (LED) and measured, with respect to the laser pulse trigger time, by means of a TDC CAEN C414 ( 25 ps/channel).

\section{Measurements and results}

Peak sensing $\left(V_{\max }\right)$, rather than charge $(Q)$ integrating ADC, was chosen because of its immunity to afterpulses, which spoils the pe resolution, as illustrated for the 3600 cells device by the chargeamplitude scatter plot of Fig. 2a. The higher quality of the $V_{\max }$ over $Q$ spectra is illustrated (besides Fig. 2a), also in the pe spectra of Fig. $2 \mathrm{~b}$.

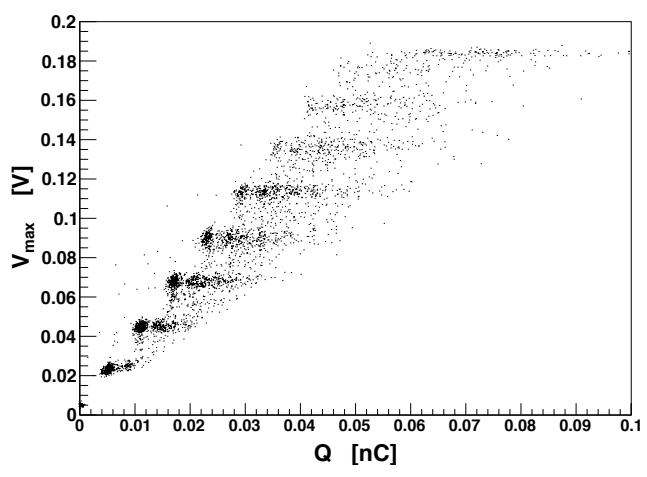

(a)
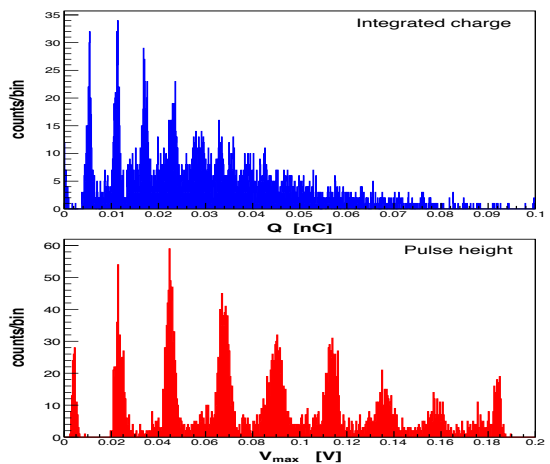

(b)

Figure 2: (a) Peak-sensing vs. charge ADC for the 3600 microcells device taken at $2.8 \mathrm{~V}$ of overvoltage. Charge (abscissa) is affected by afterpulses, while amplitude (ordinate) is immune to this background. (b) Charge and pulse height spectra corresponding to data of (a). The last peak of the pulse height histogram is due to ADC full scale. The first peak is pedestal. 
The measurements have been performed at various bias voltages and light intensities (in this work we limit ourselves to the case of the excitation of few pe's), in order to get the characteristic breakdown voltage of the two devices (Fig. 3), and to study the time resolution (timing) as a function of the overvoltage and number of $p e$ 's.

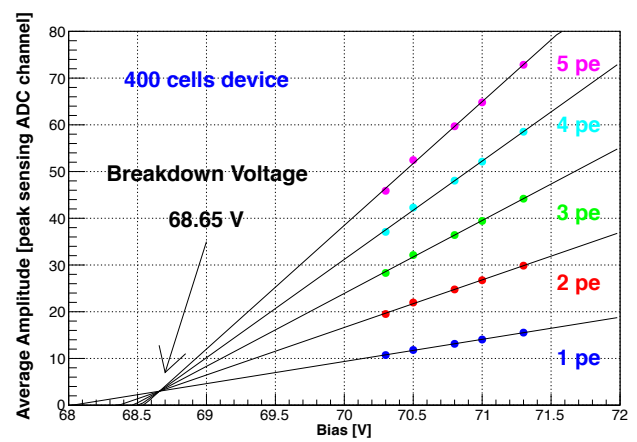

(a)

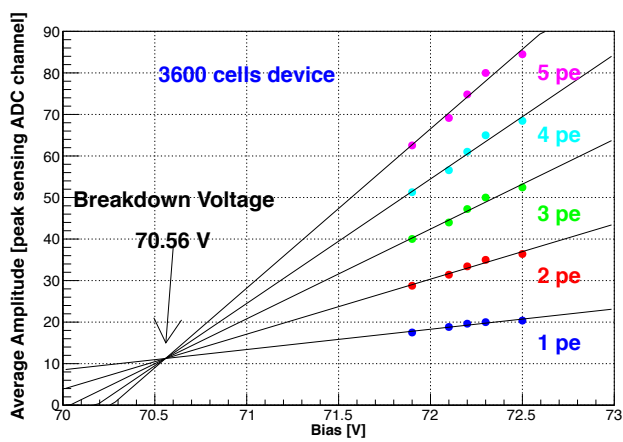

(b)

Figure 3: Linearity of the first five photopeaks versus voltage for the 400 (a) and 3600 (b) cells. Breakdown voltages are indicated.

Time resolution is measured by the width of the time distribution between the trigger time and LED output. The event-by-event analysis, owing to the time-charge (time- $V_{\max }$ ) correlation, allowed to tag the individual contribution of the $\mathrm{N}$-th $p e$ to time resolution. The time resolution as a function of number of detected simultaneous pes $N_{p e}$ follows (Fig. 4) a $1 / \sqrt{N_{p e}}$ law overlaid on a constant baseline.



(a)

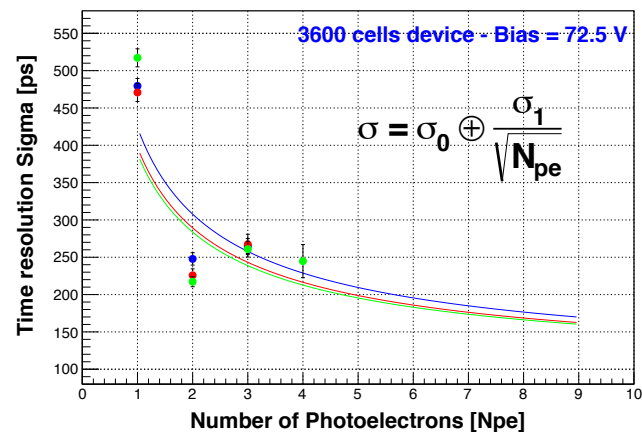

(b)

Figure 4: Time resolution vs. number of simultaneous photoelectrons at a given bias for the 400 (a) and 3600 (b) cells devices. Different colours refer to different light intensity in the range of excitation of few photoelectrons. 
If the individual pe time resolution is analyzed (Fig. 5) versus overvoltage (OV) we observe that the contribution of the first pe results dominant, with remarkable improvement from $\sigma=220$ to $\sigma=170 \mathrm{ps}$ with increasing overvoltage for the 400 cells (Fig. 4a) and from $\sigma=750$ to $\sigma=450 \mathrm{ps}$ for the 3600 cells (Fig. 4b). The positive effect of increasing gain is however counterbalanced by the increase of dark counts noise (right axes of Fig. 5), which is measured at the level of 0.5 pe, to be $0.25-0.60 \times 10^{6} \mathrm{~Hz}$ and $2.2-3.5 \times 10^{6} \mathrm{~Hz}$ for the 400 and 3600 cells sensor, respectively, increasing with the explored $\mathrm{OV}$ range.

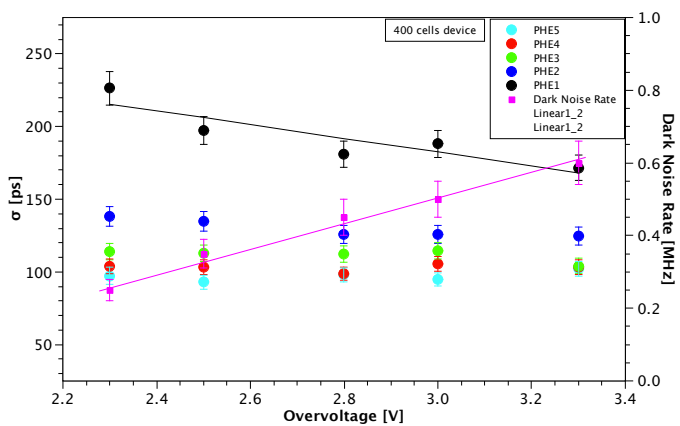

(a)

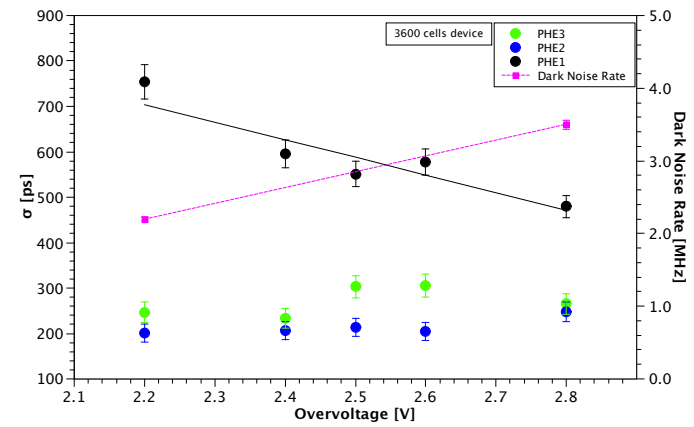

(b)

Figure 5: Time resolution of the 400 (a) and 3600 (b) cells devices for the individual photoelectrons (pe) at increasing overvoltage (gain). The measured dark noise rate is also plotted with reference to the right axes.

\section{Conclusions}

We have investigated the possible use of two types of MPPC in the focal plane of RICH detectors. At room temperature and at an overvoltage of $2.6 \mathrm{~V}$, their performance in the detection of very few photons from a very short ( $\sigma=20 \mathrm{ps}$ ) pulsed $409 \mathrm{~nm}$ laser, can be so summarized for the 400 (3600) cells MPPC's: single photoelectron timing at the level of $\sigma=200(600) \mathrm{ps}$, average dark count rate of $0.5 \times 10^{6}\left(3.0 \times 10^{6}\right) \mathrm{Hz}$, cross-talk of $14 \%(22 \%)$. This performance suggests that the two devices can be usefully employed in RICH devices. In fact, by using mechanical (or optical) funnels with an input/output area ratio of 2.5 , by limiting the time coincidence gate to $10 \mathrm{~ns}$, the two devices can distingush particles that produce a number of detected photons as low as $6(4)$ in a $1 \mathrm{~cm}$ wide annulus of $5 \mathrm{~cm}$ radius, with a signal to noise ratio of 1 . Better results can easily be obtained by lowering the temperature. If equipped with the 400 cells type, a RICH detector could even be used as timing detector.

\section{References}

[1] S. Korpar et al., Nucl. Phys. B (Proc. Suppl.) 197, 283-287 (2009)

[2] http://www.hamamatsu.com

[3] G. Collazuol et al., Nucl. Inst. Meth. A581, 461-464 (2007)

[4] http://www.alsgmbh.com/ 